\title{
Validation of Identifying Cancer Diagnosis Based on Self-Reported Information in the Japan Nurses' Health Study
}

\author{
Kota Katanoda $^{1 *}$, Yuki Ideno ${ }^{2}$, Naho Maruoka ${ }^{3}$, Kazue Nagai $^{3}$, Yoichiro Tsukada ${ }^{4}$, \\ Mei Matsuki ${ }^{4}$, Takahiro Higashi ${ }^{4}$, Kunihiko Hayashi ${ }^{3}$
}

\begin{abstract}
Background: The Japan Nurses' Health Study (JNHS) is a large-scale, nationwide prospective cohort study of female nurses. This study aimed to examine the validity of self-reported diagnosis of cancer among the JNHS cohort members $(\mathrm{N}=15,019)$. Methods: For women who reported any diagnosis of five cancers (stomach, colorectal, liver, lung and thyroid) in the biennial follow-up surveys, an additional outcome survey, medical facility survey, and confirmation of death certificate (DC) were conducted. The JNHS Validation Study Committee (referred to as "the committee") made a final decision on the reported outcomes. To examine the validity of self-reported diagnosis of cancer, the positive predictive value (PPV) was calculated using the committee's decision as the gold standard. To examine the validity of the committee's decision based on self-reports and DCs, PPV was calculated using physician-reported information as the gold standard. Results: The PPV of self-reported diagnosis in the biennial follow-up surveys was $77.8 \%$ for stomach, $66.2 \%$ for colorectal, $41.7 \%$ for liver, $60.2 \%$ for lung, and $64.6 \%$ for thyroid cancer. The corresponding PPVs in the additional outcome survey were $96.2 \%, 80.7 \%, 62.5 \%, 82.5 \%$, and $96.9 \%$, respectively. The PPV of the committee's decision was $100 \%$ for stomach, $87.5 \%$ for colorectal, $94.7 \%$ for lung, and $100 \%$ for thyroid cancer (data not available for liver cancer). The proportion of DC-only cases among committee-defined cases was below $10 \%$ for all cancers except liver cancer (28.6\%). Conclusions: The validity of identifying cancer diagnosis based on self-reported information in the JNHS was favorable for stomach, colorectal, lung and thyroid cancer.
\end{abstract}

Keywords: Cohort studies- neoplasms- validity- women's health

Asian Pac J Cancer Prev, 23 (2), 651-657

\section{Introduction}

Identifying and validating disease outcomes are some of the biggest challenges in epidemiological research. One strategy is to use information provided in physician records and to conduct periodic examinations, which is typically adopted in hospital-based studies or communitybased studies with good access to healthcare data (Boring III et al., 1996). Another way is through linkage with population-based disease registries, a strategy that is widely used for large-scale cohort studies on cancer and requires high-quality population-based cancer registry systems in the study area (Calle et al., 2002; Inoue et al., 2011; Sado et al., 2017). Alternatively, large-scale cohort studies often use self-reported disease history as a source of information due to time and cost constraints. A large number of validation studies have compared self-reported outcome information with data from objective sources, such as disease registries and medical records. For cancer or related diseases, previous findings vary by a number of factors such as study population, disease outcome and time since cancer diagnosis (Colditz et al., 1986; Kriegsman et al., 1996; Bergmann et al., 1998; Yoshinaga et al., 2001; Manjer et al., 2004; Navarro et al., 2006; Inoue et al., 2011; Cho et al., 2017; Patel et al., 2017; Cowdery et al., 2020). Validation studies conducted in Japanese cohorts from the general population have shown that sensitivity, calculated using registered cancer as the gold standard, is as low as $36 \%$ for any cancer (ranging from $8 \%$ for liver to $81 \%$ for female breast cancers), (Yoshinaga et al., 2001), although this improved to 53\% (ranging from $34 \%$ for liver to $82 \%$ for female breast cancers) in a more recent cohort (Inoue et al., 2011). By contrast, the U.S. Nurses' Health Study, a large-scale cohort of medical professionals, showed that over $90 \%$ of self-reported cancer cases were confirmed by histopathological records

${ }^{1}$ Division of Cancer Statistics Integration, Center for Cancer Control and Information Services, National Cancer Center, 5-1-1 Tsukiji Chuo-ku Tokyo, 104-0045 Japan. ${ }^{2}$ Gunma University Center for Mathematics and Data Science, 3-39-22 Showa-machi, Maebashi City, Gunma 371-8511 Japan. ${ }^{3}$ Graduate School of Health Science, Gunma University, 3-39-22 Showa-machi, Maebashi City, Gunma 371-8514 Japan. ${ }^{4}$ Division of Health Services Research, Center for Cancer Control and Information Services, National Cancer Center, 5-1-1 Tsukiji Chuo-ku Tokyo, 104-0045 Japan.*For Correspondence: kkatanod@ncc.go.jp 
(Colditz et al., 1986). The validity of self-reports for cancer identification among Japanese medical professionals has not been studied.

The Japan Nurses' Health Study (JNHS) is a large-scale, nationwide prospective cohort study of female nurses (Fujita et al., 2007; Hayashi et al., 2007). It was established by modeling the U.S. Nurses' Health Study. A total of approximately 15,000 participants were recruited for the follow-up cohort from all 47 prefectures in Japan. One of the study's primary endpoints is the incidence of cancers. The present study aimed to examine the validity of self-reported diagnosis of cancer among female nurses in the JNHS.

\section{Materials and Methods}

\section{Study design}

This validation study was conducted using postal questionnaires sent to the participants and medical facilities and information obtained from death certificates.

\section{Setting and participants}

The JNHS is an ongoing prospective cohort study of female nurses (Hayashi et al., 2007). The baseline data were collected between 2001 and 2007 from 49,927 female nurses aged 25 years or older using a self-administered questionnaire, of which 15,019 provided written informed consent to be followed-up for 10 years. Prior to this nationwide recruitment, a pilot cohort was set up in Gunma prefecture in 1999, which comprised 698 female nurses (called GNHS: Gunma Nurses' Health Study) (Maeno et al., 2005; Kato et al., 2012). The present study population was pooled from the two follow-up cohorts.

\section{Outcomes}

The JNHS and GNHS target a wide range of diseases and symptoms, with particular focus on reproductive and health outcomes common to women (Fujita et al., 2007). The present study covered cancer of the stomach, colon/ rectum, liver, lung and thyroid. Other outcomes including cancer of the breast and female genital organs will be examined elsewhere.

\section{Baseline and follow-up surveys}

The baseline questionnaire was distributed with an invitation letter; respondents completed the questionnaire and mailed it back to the JNHS office with a signed form providing informed consent. The questionnaire included items on lifestyle, drug use, physical and reproductive condition, and history of disease. Participants were followed up for 10 years. Follow-up questionnaires inquiring about changes in lifestyle, other exposure items, and incidence of disease were sent every 2 years. For the baseline and follow-up surveys, except the 2-year follow-up, history or incidence of each disease was asked using the question "Have you ever been diagnosed with a disease?" This was followed by a question about the respondent's age at diagnosis. In the 2-year follow-up survey, the question "Have you been diagnosed with a disease during the past two years?" was used, followed by a question inquiring about the year and month of the diagnosis. Details of the survey design are provided in our previous report (Hayashi et al., 2007).

\section{Validation}

To validate of self-reported diagnosis of diseases identified in the biennial follow-up surveys, an additional outcome survey, medical facility survey, and confirmation of death certificate were conducted (Figure 1). Women eligible for these validation checks were cohort members who completed at least one of the routine biennial follow-up surveys $(\mathrm{N}=15,019$ for JNHS and $\mathrm{N}=698$ for GNHS) and who did not report a history of diagnosis of each outcome at baseline. First, for each outcome, we defined the self-reported answer to the question about disease diagnosis in the biennial follow-up surveys as "definition l". Second, the additional outcome survey questionnaire was sent to participants who answered "yes" to definition 1. This additional survey was conducted separately for each outcome (i.e. each type of cancer in the present study), and included the following question items (examples for stomach cancer):

"Have you ever been diagnosed with stomach cancer by a medical doctor?" (yes or no)

"When were you diagnosed?" (year and month)

"How were you diagnosed?" (answer chosen from a list: screening, abdominal discomfort, stomach upset, etc.)

"Which test(s) did you undergo for the diagnosis?" (answer chosen from a list: upper gastrointestinal endoscopy, X-ray, Helicobacter pylori test, etc.)

"Were you hospitalized for the diagnosis and/or treatment?" (yes or no)

"What type of treatment(s) did you receive?" (answer chosen from a list: gastric resection with open surgery, laparoscopic gastrectomy, endoscopic surgery, etc.)

The self-reported diagnosis indicated in the additional survey was defined as "definition 2".

Third, the medical facility survey was conducted among participants who provided written informed consent to participate in this survey and the contact information of the medical doctor in charge of their case. A questionnaire was sent to the provided postal address of the medical facility inquiring about the following items regarding the patient (examples for stomach cancer): date of diagnosis, primary / recurrent / multiple cancer, smoking history, family history of stomach cancer, height and weight, modality of diagnosis (histology, cytology, imaging, clinical, and/or other), primary site (upper, middle, lower, esophagogastric junction), tumor size, invasion into lymph nodes or veins, clinical and pathological TNM (Tumor, lymph Nodes, and Metastasis) classifications, site of metastasis, if any. The physicianreported diagnosis provided in the medical facility survey was defined as "definition 3".

Death certificate confirmation is routinely conducted in the JNHS. Individual records of death certificates were obtained from the Office of Vital Statistics of Japan under official approval, and linked to the personal identification data of the cohort. This linkage was performed using date of birth, date of death, and municipality of residence data as key variables.

Combining the information obtained from the four 
sources (definitions 1-3 and death certificate confirmation), the JNHS Validation Study Committee (referred to as "the committee") made a final decision on the incidence of the reported outcome (definition 4). In cases where there was no physician-reported diagnosis (definition 3), the committee made their decision (definition 4') based on three information sources (definitions 1-2 and death certificate confirmation). The majority of the incident cases were decided upon definition 4'.

The primary aim of the present study was to examine the validity of self-reported diagnosis of cancer using the following indices (Figure 1):

A. Positive predictive value (PPV) of definition 1 using definition 4 as the gold standard

B. PPV of definition 2 using definition 4 as the gold standard

C. Proportion of death certificate-only cases among diagnosed cases defined by the committee's overall decision (definition 4) (i.e. potentially false-negative cases in the self-reporting scheme)

The secondary aim was to examine the validity of the committee's overall decision on cancer diagnosis made based on self-reported diagnosis and death certificate confirmation (definition 4') using physician-reported information as the gold standard (definition 3). Specifically, the following indices were calculated:

D-1. PPV of definition 4' using definition 3 as the gold standard

D-2. Negative predictive value (NPV) of definition 4' using definition 3 as the gold standard (calculated only for cases identified as "positive" in self-reported sources)

The GNHS study protocol was approved by the institutional review board of Gunma University, Japan (Approval No. 3, 1999), and the JNHS study protocol was approved by the institutional review board of Gunma University, Japan (Approval No. 101, 2001) and by the ethics review board of Japan's National Institute of Public Health, Japan (Approval No. 03007, 2003). Informed consent was obtained from all individual participants included in the study.

\section{Results}

Table 1 shows the basic characteristics of the participants of the JNHS and its pilot study, the GNHS. Median age of the cohort members was 41 and 37 years old in the JNHS and GNHS, respectively. Women aged $30-49$ years accounted for $77 \%$ of the JNHS and $59 \%$ of the GNHS cohort, and the median follow-up period was 14 and 18 years, respectively.

Table 2 shows the results of the outcome surveys. The percentage of potential incident cases identified through the biennial follow-up surveys (among all eligible women) was $0.5 \%$ for stomach, $0.9 \%$ for colorectal, $0.1 \%$ for liver, $0.6 \%$ for lung, and $0.7 \%$ for thyroid cancer. After excluding cases due to withdraw, loss to follow-up, and/or death, over $90 \%$ of potential incident cases were subjected to the additional outcome survey. We did not calculate response rates to the biennial follow-up surveys because the cohort members provided responses to at least one of the routine biennial follow-up surveys.

In the additional outcome survey, the response rate ranged from $81.7 \%$ to $91.4 \%$. Almost all respondents re-reported their diagnosis $(82.8 \%$ to $93.2 \%)$. Among the respondents of the additional outcome survey, half or less agreed to participate in the medical facility survey (50.9\% for stomach, $45.0 \%$ for colorectal, $25.0 \%$ for liver, $50.9 \%$ for lung, and $57.8 \%$ for thyroid cancer). The medical facility survey revealed good agreement between self-reported and physician-reported diagnoses (100.0\% for stomach, $97.0 \%$ for colorectal, $86.4 \%$ for lung, and $96.9 \%$ for thyroid cancer) (there were no respondents for liver cancer). The number of deaths identified through death certificates (death certificate-only cases) was 7 (2) for stomach, 6 (3) for colorectal, 2 (2) for liver, 10 (4) for lung, and 2 (0) for thyroid cancer. Finally, the committee confirmed the diagnosis of 58 stomach, 93 colorectal, 7 liver, 54 lung, and 64 thyroid cancer cases, corresponding to $80.6 \%, 68.4 \%, 58.3 \%, 65.1 \%, 64.6 \%$ of the potential incident cases in the biennial follow-up

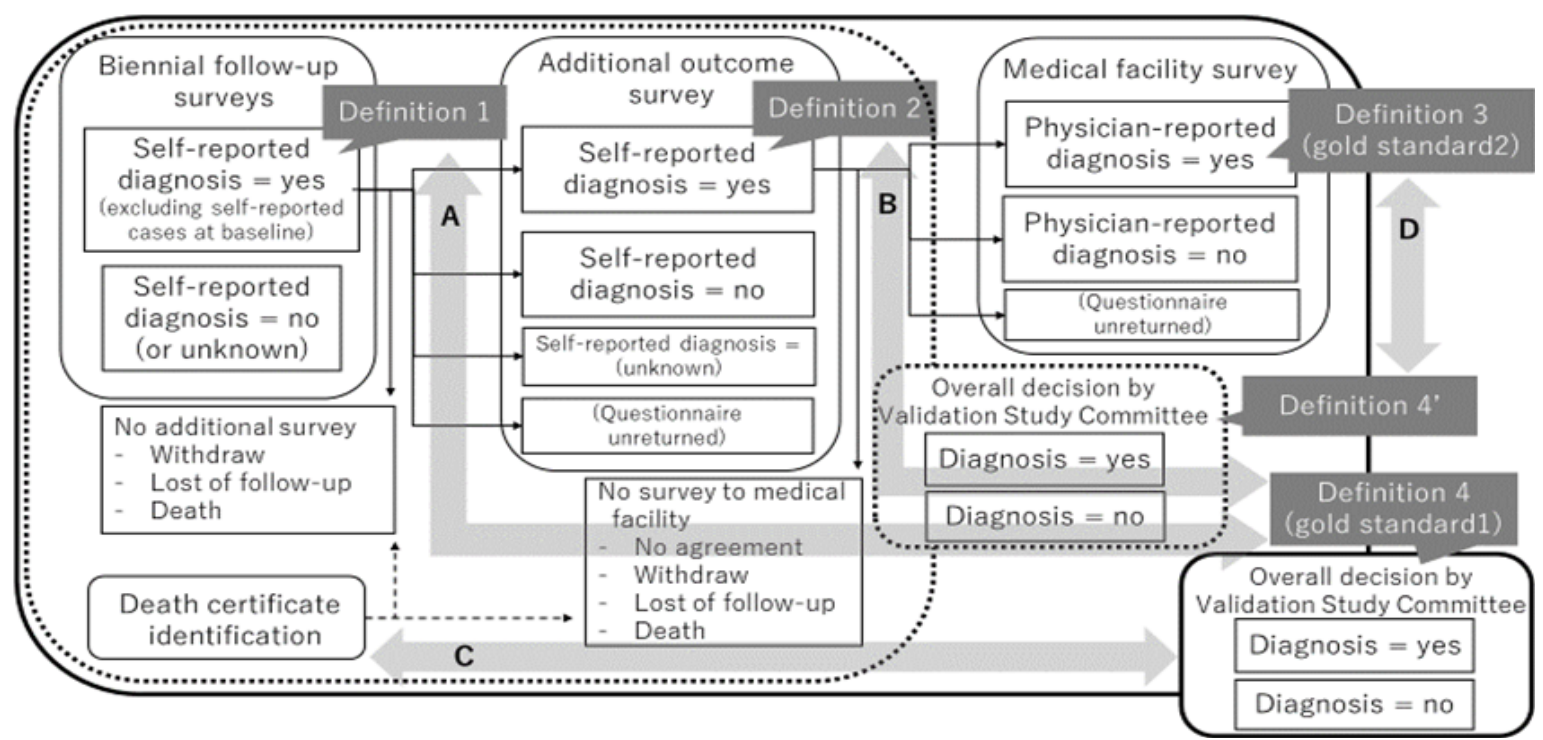

Figure 1. Flow Chart of the Outcome Surveys. The flow of the outcome surveys in the Japan Nurses' Health Study and the comparison diagrams for this validation study are illustrated. 
Table 1. Basic Characteristics of the Japan and Gunma Nurses' Health Studies

\begin{tabular}{|c|c|c|c|c|}
\hline & \multicolumn{2}{|c|}{ JNHS } & \multicolumn{2}{|c|}{ GNHS } \\
\hline Total number & \multicolumn{2}{|c|}{15,019} & \multicolumn{2}{|r|}{698} \\
\hline \multicolumn{5}{|l|}{ Age at baseline } \\
\hline Mean (SD) & 41.8 & (8.2) & 37.3 & $(10.1)$ \\
\hline Median (Q1-Q3) & 41 & $(35-48)$ & 37 & $(28-45)$ \\
\hline$<30$ years & 498 & $(3.3 \%)$ & 194 & $(27.8 \%)$ \\
\hline $30-34$ years & 2859 & $(19.0 \%)$ & 96 & $(13.8 \%)$ \\
\hline $35-39$ years & 3061 & $(20.4 \%)$ & 115 & $(16.5 \%)$ \\
\hline $40-44$ years & 3026 & $(20.1 \%)$ & 107 & $(15.3 \%)$ \\
\hline $45-49$ years & 2672 & $(17.8 \%)$ & 95 & $(13.6 \%)$ \\
\hline $50-54$ years & 1959 & $(13.0 \%)$ & 53 & $(7.6 \%)$ \\
\hline 55-59 years & 764 & $(5.1 \%)$ & 33 & $(4.7 \%)$ \\
\hline $60-64$ years & 138 & $(0.9 \%)$ & 5 & $(0.7 \%)$ \\
\hline $65+$ years & 42 & $(0.3 \%)$ & 0 & $(0.0 \%)$ \\
\hline \multicolumn{5}{|l|}{ Follow-up years } \\
\hline Mean (SD) & 11.9 & (4.4) & 14.3 & (5.3) \\
\hline Median (Q1-Q3) & 14 & $(10-16)$ & 18 & $(10-18)$ \\
\hline
\end{tabular}

JNHS, Japan Nurses' Health Study; GNHS, Gunma Nurses' Health Study; SD, standard deviation; Q1, 25 percentile; Q3, 75 percentile

surveys, respectively. The percentage of all eligible women confirmed to have a diagnosis by the committee was $0.4 \%$ for stomach, $0.6 \%$ for colorectal, $0.05 \%$ for liver, $0.4 \%$ for lung, and $0.4 \%$ for thyroid cancer.

Table 3 shows the results of the PPV and other validation indices. The PPV of self-reported diagnosis in the biennial follow-up surveys was highest for stomach $(77.8 \%)$ and lowest for liver $(41.7 \%)$, and intermediate for colorectal $(66.2 \%)$, lung $(60.2 \%)$, and thyroid (64.6\%) cancer. The PPV of self-reported diagnosis in the additional outcome survey was also lowest for liver cancer $(62.5 \%)$, while the highest values were observed for stomach $(96.2 \%)$ and thyroid $(96.9 \%)$ cancer. The proportion of death certificate-only cases among diagnosed cases defined by the committee's overall decision was less than $10 \%$ for all cancers except liver cancer $(28.6 \%)$. The PPV of the overall decision compared to the physician-reported diagnosis was as high as $87.5 \%$ for colorectal cancer and even higher for stomach (100\%), lung (94.7\%), and thyroid (100\%) cancer (the PPV could not be calculated for liver cancer because there were no respondents). The NPV of the overall decision compared to the physician-reported diagnosis was $100 \%$ for colorectal and lung cancer and $0 \%$ for thyroid cancer, although the number of cases available to calculate this index was very small. Corresponding results from the GNHS are shown in Supplementary Tables 1-2.

\section{Discussion}

The present study examined the validity of selfreported diagnosis of cancer among female nurses in the JNHS by combining information from the participants medical facilities, and death certificates. We observed favorable PPVs for stomach, colorectal, lung, and thyroid cancers (approximately $60-80 \%$ using the committee's overall decision based on the additional outcome survey and death certificate confirmation as the gold standard). For these outcomes, death certificate-only cases, which can be considered false-negative cases, were as low as $10 \%$, and PPVs calculated for a sub-sample using information obtained from medical facilities as the gold standard were over $87 \%$. Our results indicate that the validity of identifying cancer diagnosis based on selfreported information in the JNHS was favorable for stomach, colorectal, lung, and thyroid cancer.

The PPVs of self-reported diagnosis of cancer in this study were higher than those reported in a populationbased cohort study of community-dwelling populations. In a validation study of the Japan Public Health Centerbased Prospective Study (JPHC Study), PPVs calculated by comparison with cancer registry data were $52 \%$ for stomach, $47 \%$ for colorectal, $46 \%$ for lung, $58 \%$ for female breast cancer.(Inoue et al., 2011) This discrepancy in PPV values may be due to differences in the cohort profiles: our participants were female nurses, who may have provided more accurate responses about their health conditions than women in the general population. Consistent with our hypothesis, the original U.S. Nurses' Health Study demonstrated the accuracy of self-reported outcomes from female nurses.(Colditz et al., 1986)

The validity of self-reported diagnosis of liver cancer was relatively low in our study: the PPV (vs. the committee's overall decision) and proportion of death certificate-only cases were approximately $40 \%$ and $30 \%$, respectively (the PPV vs. information from medical facilities could not be calculated). This result was consistent with those of previous studies. In the JPHC Study cited above, the PPV was $31 \%$ for liver cancer. (Inoue et al., 2011) An earlier validation study from the same JPHC group also reported a very low sensitivity for self-reported liver cancer.(Yoshinaga et al., 2001) The inaccuracy of self-reported liver cancer may be due to the smaller number of cases (liver cancer: 12 cases, colorectal cancer: 136 cases in the present study). Unfavorable prognosis of this cancer may also be a contributing factor, which was reflected in the higher proportion of death certificate-only cases $(29 \%)$.

The biennial follow-up surveys, additional outcome survey, medical facility survey, and death certificate confirmation are conducted as part of the JNHS's routine follow-up process. In the present study, PPVs of selfreports using the committee's overall decision as the gold standard were higher in the additional outcome survey than in the biennial follow-up surveys (B vs. A in Table 3). This is a matter of course because the overall decision was based on detailed clinical information obtained from the additional outcome survey. However, from a quality assurance perspective on the routine follow-up, this additional survey, which received response rates of over $80 \%$, is useful for improving the accuracy of outcome information in our study. Additionally, we identified $3-7 \%$ of cancer cases (other than liver cancer) from death certificates, suggesting that death certificate confirmation is useful for supplementing cases that cannot be identified by self-report.

Use of physician records data is an ideal way to 

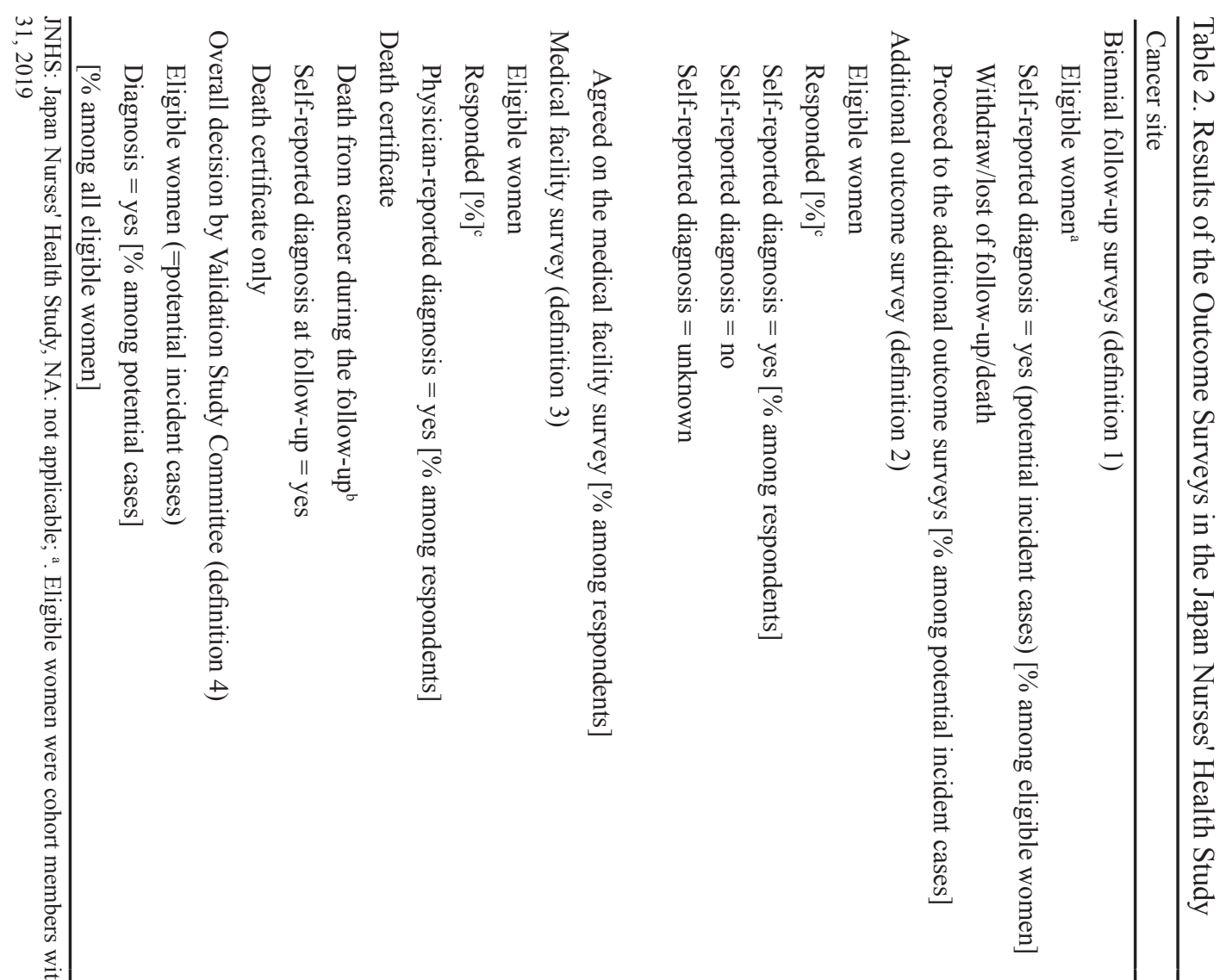

m.

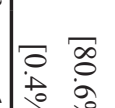

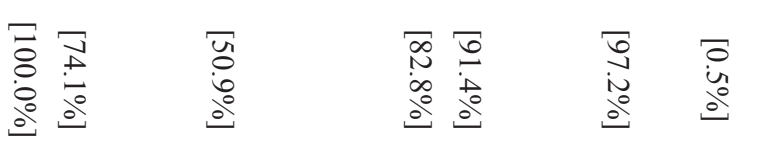

๖
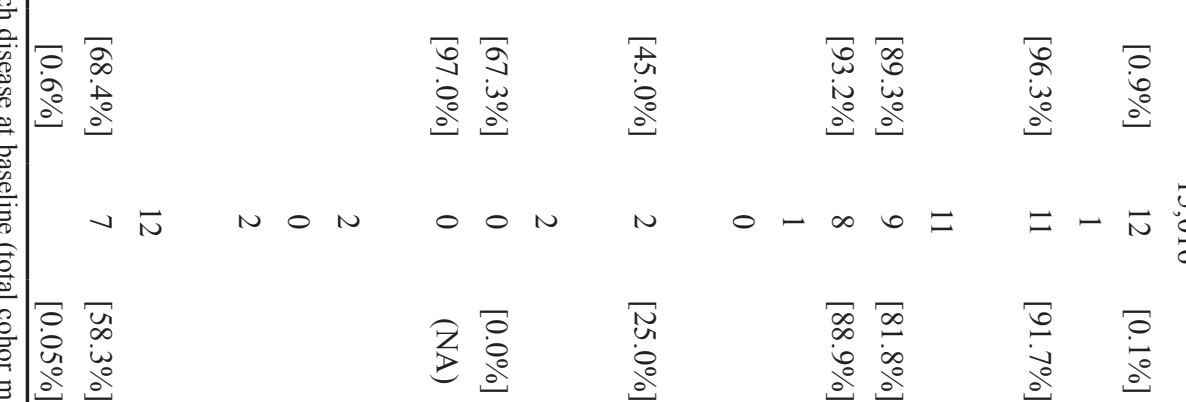

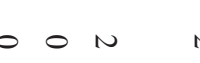

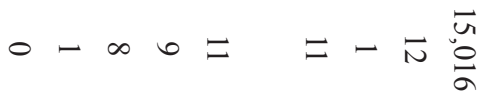

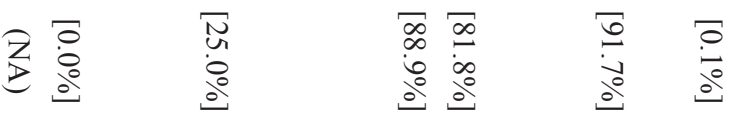

น

党

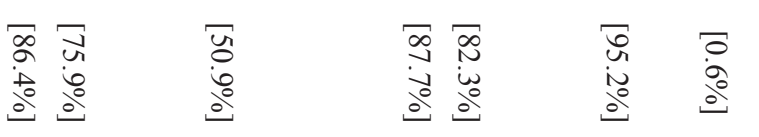

象

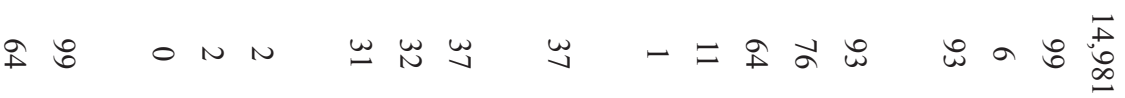

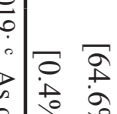

ॠ

$\bar{u}$
$\infty$
$\vdots$
0

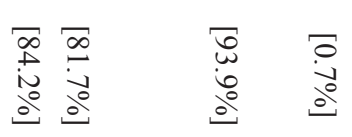




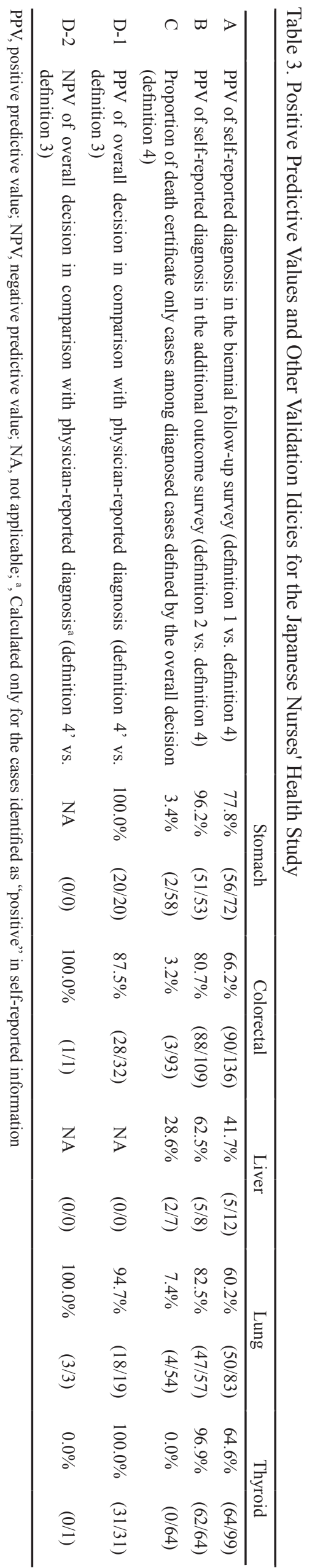

confirm self-reported disease outcomes. While this is the main strategy adopted by the U.S. Nurses' Health Study, in which they contact each patient to obtain permission and the patients' hospitals to obtain copies of pertinent records,(Colditz and Hankinson, 2005) other studies obtained medical records only from a sub-sample of cases.(Hunter et al., 1992) Studies examining cancer outcomes have generally been successful at obtaining medical records from a high percentage of reported cases (e.g. 93-94\%).(Colditz et al., 1995; Feskanich et al., 2007) Although the JNHS adopts the same method, the proportion of self-reported cases providing their hospitals' contact information has been much lower; the proportion ranged from $25.0 \%$ (liver cancer) to $57.8 \%$ (thyroid cancer) (Table 2). Furthermore, the response rate from medical facilities was also low, ranging from $0.0 \%$ (liver cancer) to $86.5 \%$ (thyroid cancer). Therefore, although there was good agreement between self-report and medical facilities data ( $86.4 \%$ or higher), this method of confirmation was only feasible for a portion of cases. While it may be possible to increase the proportion of medical facility-confirmed cases by calling the hospitals or using other reminder methods, this requires financial and human resources. Based on the fact that we found good agreement between self-report and medical facilities data, we think that contacting medical facilities for a sub-sample of self-reported cases (i.e. participants who provided their hospitals' contact information) is a feasible and valid method for the JNHS study.

One of the strengths of the present study is the high response rates in the outcome surveys at least from the participants. We were also able to obtain comprehensive information from death certificates. Nevertheless, there are several limitations in our study. First, we did not examine the sensitivity or specificity of the self-reported outcomes. To do this, we would need high-quality population-based cancer registry data from the study areas. Although population-based cancer registry data were available in all 47 prefectures for cancer cases diagnosed in 2012 and later, not all of them met the standard quality criteria.(Hori et al., 2015) Because our cohort members were recruited from all over Japan, it was difficult to obtain high-quality cancer registry data for all members. The National Cancer Registry was started in 2016 in Japan based on the Act on Promotion of Cancer Registries.(Matsuda and Sobue, 2015; Tanaka and Matsuda, 2015) This new data source allows us to calculate the sensitivity and specificity for cases diagnosed in 2016 and later.

Second, some of the information sources were not independent of each other. Specifically, the decision of the committee, one of the gold standards was based on the results of the follow-up and outcome surveys, which were also used for comparisons. However, we also conducted comparisons between independent sources for a sub-sample, namely, the biennial follow-up and additional outcome surveys and information from the medical facilities survey.

In conclusion, the validity of identifying cancer diagnosis based on self-reported information in the JNHS was favorable for stomach, colorectal, lung and thyroid 
cancers.

\section{Author Contribution Statement}

KK wrote the manuscript, $\mathrm{KH}$ supervised the whole study, YI, NM, and KN collected and analyzed the data, and YT, MM, and TH examined the clinical data.

\section{Acknowledgements}

The authors appreciate the cooperation of all the nurses who participated in the present study and the JNHS. The authors are also grateful to all the staff members of the JNHS for assistance with study coordination and data management.

\section{Availability of Data}

The tabulated data that support the findings of this study are available from the last author $(\mathrm{KH})$ upon reasonable request. The raw data are not publicly available because providing such data was not included in the informed consent.

\section{Funding Statement}

This work was supported by the Japan Society for the Promotion of Science [grant number 18H04069 to $\mathrm{KH}]$ and the Japan Agency for Medical Research and Development [grant number 21gk0210027h0001 to KH].

\section{Potential Conflict of Interest}

KK, YI, and KN received a JMWH Bayer Grant (1 million JPY) via the Japan Society for Menopause and Women's Health from Sep. 2017 to Aug. 2019, from Sep. 2019 to Aug. 2020, and from Sep. 2020 to Aug. 2021, respectively. None for the other authors.

\section{References}

Bergmann MM, Calle EE, Mervis CA, et al (1998). Validity of self-reported cancers in a prospective cohort study in comparison with data from state cancer registries. $\mathrm{Am} \mathrm{J}$ Epidemiol, 147, 556-62.

Boring III JR, Daniels SR, Eley JW, et al (1996). Cohort studies. In 'Medical Epidemiology, Third Edition', Eds The McGraw-Hill Companies, Inc., U.S.A., PP 113-26.

Calle EE, Rodriguez C, Jacobs EJ, et al (2002). The American Cancer Society Cancer Prevention Study II Nutrition Cohort: rationale, study design, and baseline characteristics. Cancer, 94, 2490-501.

Cho S, Shin A, Song D, et al (2017). Validity of self-reported cancer history in the health examinees (HEXA) study: A comparison of self-report and cancer registry records. Cancer Epidemiol, 50, 16-21.

Colditz GA, Hankinson SE (2005). The Nurses' Health Study: lifestyle and health among women. Nat Rev Cancer, 5, 388-96.

Colditz GA, Hankinson SE, Hunter DJ, et al (1995). The use of estrogens and progestins and the risk of breast cancer in postmenopausal women. $N$ Engl J Med, 332, 1589-93.

Colditz GA, Martin P, Stampfer MJ, et al (1986). Validation of questionnaire information on risk factors and disease outcomes in a prospective cohort study of women. $\mathrm{Am} \mathrm{J}$ Epidemiol, 123, 894-900.
Cowdery SP, Stuart AL, Pasco JA, et al (2020). Validity of selfreported cancer: Comparison between self-report versus cancer registry records in the Geelong Osteoporosis Study. Cancer Epidemiol, 68, 101790.

Feskanich D, Bain C, Chan AT, et al (2007). Aspirin and lung cancer risk in a cohort study of women: dosage, duration and latency. Br J Cancer, 97, 1295-9.

Fujita T, Hayashi K, Katanoda K, et al (2007). Prevalence of diseases and statistical power of the Japan Nurses' Health Study. Ind Health, 45, 687-94.

Hayashi K, Mizunuma H, Fujita T, et al (2007). Design of the Japan Nurses' Health Study: a prospective occupational cohort study of women's health in Japan. Ind Health, 45, 679-86.

Hori M, Matsuda T, Shibata A, et al (2015). Cancer incidence and incidence rates in Japan in 2009: a study of 32 populationbased cancer registries for the Monitoring of Cancer Incidence in Japan (MCIJ) project. Jpn J Clin Oncol, 45, 884-91.

Hunter DJ, Colditz GA, Stampfer MJ, et al (1992). Diet and risk of basal cell carcinoma of the skin in a prospective cohort of women. Ann Epidemiol, 2, 231-9.

Inoue M, Sawada N, Shimazu T, et al (2011). Validity of selfreported cancer among a Japanese population: recent results from a population-based prospective study in Japan (JPHC Study). Cancer Epidemiol, 35, 250-3.

Kato C, Shimada J, Hayashi K (2012). Sleepiness during shift work in Japanese nurses: A comparison study using JESS, SSS, and actigraphy. Sleep Biological Rhythms, 10, 109-17.

Kriegsman DM, Penninx BW, van Eijk JT, et al (1996). Self-reports and general practitioner information on the presence of chronic diseases in community dwelling elderly. A study on the accuracy of patients' self-reports and on determinants of inaccuracy. J Clin Epidemiol, 49, 1407-17.

Maeno T, Ohta A, Hayashi K, et al (2005). Impact of reproductive experience on women's smoking behaviour in Japanese nurses. Public Health, 119, 816-24.

Manjer J, Merlo J, Berglund G (2004). Validity of self-reported information on cancer: determinants of under- and overreporting. Eur J Epidemiol, 19, 239-47.

Matsuda T, Sobue T (2015). Recent trends in population-based cancer registries in Japan: the Act on Promotion of Cancer Registries and drastic changes in the historical registry. Int J Clin Oncol, 20, 11-20.

Navarro C, Chirlaque MD, Tormo MJ, et al (2006). Validity of self reported diagnoses of cancer in a major Spanish prospective cohort study. J Epidemiol Community Health, 60, 593-9.

Patel AV, Jacobs EJ, Dudas DM, et al (2017). The American Cancer Society's Cancer Prevention Study 3 (CPS-3): Recruitment, study design, and baseline characteristics. Cancer, 123, 2014-24.

Sado J, Kitamura T, Kitamura Y, et al (2017). Rationale, design, and profile of the Three-Prefecture Cohort in Japan: A 15year follow-up. J Epidemiol, 27, 193-9.

Tanaka H, Matsuda T (2015). Arrival of a new era in Japan with the establishment of the Cancer Registration Promotion Act. Eur J Cancer Prev, 24, 542-3.

Yoshinaga A, Sasaki S, Tsugane S (2001). Sensitivity of selfreports of cancer in a population-based prospective study: JPHC Study Cohort I. J Clin Epidemiol, 54, 741-6.

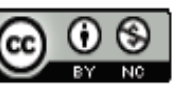

This work is licensed under a Creative Commons AttributionNon Commercial 4.0 International License. Asian Pacific Journal of Cancer Prevention, Vol 23 